\title{
The revolution of the Earth around the Sun causes the vortex-induced vibration of south-north trending bridges and impacts seismic activities
}

Xiaoyong $\mathrm{Lu}^{2}$, Haili Ran ${ }^{2}$, Ruohan Zheng ${ }^{3}$, Ting Zeng ${ }^{3}$, Cui Yang ${ }^{1}$, Qiuyun Liu ${ }^{1, *}$

${ }^{1}$ School of Life Sciences, Sun Yat-sen University, Guangzhou 510275, China.

${ }^{2}$ School of Marine Sciences, Sun Yat-sen University, Guangzhou 510275, China.

${ }^{3}$ School of Chemistry, Sun Yat-sen University, Guangzhou 510275, China

${ }^{*}$ Corresponding author: Qiuyun Liu, Ph.D, School of Life Sciences, Sun Yat-sen University, Guangzhou 510275, China.E-mail: 1sslqy@mail.sysu.edu.cn 


\begin{abstract}
:
The vortex-induced vibration in east-west trending Humen Bridge in Guangdong, China is related to the self-rotation of the Earth, whereas the south-north trending Wuhan Parrot Island Bridge is more likely to be triggered by the revolution of the Earth around the Sun. In the early summer, the northern bank of Yangtze River is subjected to solar pull and decelerates more slowly towards the river with low density water. In late summer, the southern bank of the Yangtze River decelerates more slowly towards the river. These factors jointly contribute to the shortening of the distance between the piers of the bridge and trigger shaking of the bridge. Seismic activities are influenced by both self-rotation and revolution of the Earth.
\end{abstract}

\title{
Keywords:
}

vortex-induced vibration; south-north trending; Wuhan Parrot Island Bridge; potential energy; low density water; slower deceleration of larger mass 
Recently, the shaking of south-north trending Wuhan Parrot Island Bridge in Hubei, China causes concern among the public. Due to the presence of potential energy on the surface of Earth, slower decelerations of large mass or/and faster accelerations of smaller mass bring about earthquake, volcanoes, sand and dust storms, petroleum fields, hot springs, etc. ${ }^{1-11}$

The vortex-induced vibration in east-west trending Humen Bridge in Guangdong, China is related to the self-rotation of the Earth, whereas the south-north trending Wuhan Parrot Island Bridge is more likely to be triggered by the revolution of the Earth around the Sun. ${ }^{11-12}$ In the early summer, the northern bank of Yangtze River is subjected to solar pull and decelerates more slowly towards the river. In late summer, the southern bank of the Yangtze River decelerates more slowly towards the river. Collectively, these forces shorten the distance between the piers of the bridge and trigger shaking of the bridge.

Likewise, the northeastward drifting of Tibetan plateau is triggered by both the self-rotation and revolution of the Earth ${ }^{11}$, leading to the drifting to the east and to the north respectively, which causes intensive and extensive seismic activities in Sichuan Province, China.

Other types of bridges with non-strict south-north or east-west trending are influenced by both the self-rotation and revolution of the Earth.

\section{Conflict of interest statement}

The authors declare no conflict of interests.

\section{Funding}

This work was supported by grants from the Science and Technology Transformation Program of Sun Yat-sen University of China No. 33000-18843234 and Guangzhou Science and Technology Program No. 201804010328 to Q. Liu.

\section{Acknowledgements}

We thank Yan Shi for language editing.

\section{References}

1. Yan S, Du Y, Liu Q, et al. Filtering out non-specific animal behavior to better predict impending earthquakes. OSF Preprints. 2020. https://doi.org/10.31219/osf.io/bs3qd

2. Yan S, Li J, Liu Q. Earthquakes-an intricate trio dance of gravitation. Science 2018; http://science.sciencemag.org/content/354/6315/1027/tab-e-letters.

3. $\mathrm{Xu} \mathrm{H}, \mathrm{Wu}$ J, Liu Q. Earth's self-rotation: high-velocity movements manifested in the solar and lunar gravitational fields. Science 2019; https://science.sciencemag.org/content/354/6308/88/tab-e-letters.

4. He R, He S, Nuertai X, et al. The faster acceleration of smaller masses in the west of Americas. Science 2017; http://science.sciencemag.org/content/354/6315/1027/tab-e-letters.

5. Liu Q, Gao J, Pan W, et al. Differentia in decelerations of masses: when the Earth 
meets lunar and solar pulls. Science 2017; http://science.sciencemag.org/content/354/6315/1027/tab-e-letters.

6. Zhu X, Yang F, Li Z, Liu Q. Why the Indian subcontinent drifts toward Eurasia. Science, 2017; http://science.sciencemag.org/content/356/6340/841/tab-e-letters

7. Wan Y, Shi Y, Li Z, et al. How focal depth is generated? Science 2017; http://science.sciencemag.org/content/354/6315/1027/tab-e-letters.

8. Yan S, Wang X, Qi J, Liu Q. Volcanoes or earthquakes: Wrist wrestling. Science, 2019; http://science.sciencemag.org/content/358/6370/1520/tab-e-letters.

9. Yan $\mathrm{S}, \mathrm{Xu} \mathrm{X}$, Chen L, et al. Earthquakes: The rocking cradle. Science 2019; https://science.sciencemag.org/content/354/6315/1027/tab-e-letters.

10. Zhong $\mathrm{H}$, Wang $\mathrm{K}$, Shi $\mathrm{Y}$, et al. Tsunamis is a prime example of the vertical component of the forces during earthquakes. Science 2017; http://science.sciencemag.org/content/358/6367/1164/tab-e-letters

11. Zheng R, Jin W, Zeng T, et al. The Uplift of the Qinghai-Tibet Plateau: The Driver Behind the Scene. OSF Preprints 2020. DOI: 10.31219/osf.io/hpvk7. https://osf.io/hpvk7

12. Ran H, Lu X, Zheng R, et al. 2020. Vortex-induced Vibration: The Slower Deceleration of the West Bank Leads to the Shortening of the Humen Bridge in Guangdong, China. OSF Preprints 2020. doi:10.31219/osf.io/3t4a6. https://osf.io/3t4a6 